\title{
El personal científico del CENIM frente al reto de las publicaciones científicas: los años 1995-1999(*)
}

\author{
F.J. Alguacil ${ }^{*}$ M. Carsî* y O.A. Ruano*
}

Resumen $\quad$ En este trabajo se analiza la labor del personal investigador del Centro Nacional de
Investigaciones Metalúrgicas (CENIM) en cuanto a publicaciones correspondientes al
período 1995-99.

Palabras clave Metalurgia. Publicaciones.

\section{The response scientific of the staff of CENIM the scientific publications challenge of the 1995-1999 years}

\author{
Abstract The present work analyses the work carried out by the scientific staff of CENIM regarding \\ the publications in the period 1995-99. \\ Keywords Metallurgy. Publications.
}

\section{INTRODUCCIÓN}

En 1992, el Presidente del Consejo Superior de Investigaciones Científicas nombró un Comite Internacional compuesto por los Profs. Dres. Alvarez, Cahn, Domínguez, Ehrlich y Kubin para llevar a cabo una "evaluación, especialmente de la actividad científica", del CENIM. En el informe presentado al Presidente del CSIC por este Comité se recomendó una fuerte reorientación en la actividad del Centro (de su personal investigador principalmente), de manera que se aumentara la cantidad y, sobre todo, la calidad de la investigación científica (y tecnológica). Uno de los objetivos debía ser aumentar el número de publicaciones científicas preferentemente en revistas recogidas en el JCR (Journal Citation Reports).

El personal investigador del CENIM, una vez asimiladas estas recomendaciones y objetivos, aportó su esfuerzo para conseguir esta "nueva reorientación". En este trabajo se hace un balance de los resultados logrados en el periodo 1995-1999 referente a las publicaciones científicas en revistas incluidas en el JCR.

\section{EL PERSONAL}

Debe considerarse que el potencial científico de un Centro de Investigación tipo del CSIC lo representa el personal investigador en plantilla adscrito al mismo. Al fin y al cabo este personal es el encargado de llevar a cabo el día a día en la investigación, aunque existen actualmente otros condicionamientos (p. ej.: la búsqueda y solicitud más o menos incesante de fondos para investigar, asistencia a reuniones, etc.) que están desvirtuando el trabajo primordial del investigador, que no es otro que el de investigar en el laboratorio o, en su caso, en la investigación de campo.

Con estas consideraciones, ¿cual es la realidad del CENIM en cuanto a personal científico a lo largo de estos años?. La tabla I muestra cuál ha sido esta evolución en el quinquenio analizado. Se observa que la plantilla de investigadores del CENIM ha tenido un crecimiento cero, por lo que se pueden considerar dos puntos de vista: uno, no ha disminuido el potencial científico del CENIM (las nuevas incorporaciones han servido para cubrir las bajas generadas por diversos motivos, pero eso sí, la

(•) Trabajo recibido el día 10 de Octubre de 2001 y aceptado en su forma final el día 1 de Noviembre de 2001.

$\left(^{*}\right)$ Centro Nacional de Investigaciones Metalúrgicas (CENIM), Consejo Superior de Investigaciones Científicas (CSIC), Avda. Gregorio del Amo, 8. 28040 Madrid (España). 
Tabla I. Total de investigadores en plantilla por año Table I. Total scientific staff per year

\begin{tabular}{lc}
\hline Año & Número \\
1995 & 43 \\
1996 & 42 \\
1997 & 43 \\
1998 & 42 \\
1999 & 42 \\
\hline
\end{tabular}

*Se consideran en esta tabla y a lo largo del trabajo: Profesores de Investigación, Investigadores Científicos y Científicos Titulares.

edad media de los investigadores en plantilla va en aumento); dos, realmente existe un verdadero estancamiento en la plantilla de investigadores del CENIM, que hace temer que algunas de las líneas de investigación (productivas desde el punto de vista de lograr fondos para investigar y en la generación de publicaciones científicas y tecnológicas) puedan verse colapsadas a medio plazo.

\section{LOS ARTÍCULOS CIENTÍFICOS}

Este personal científico, ¿como ha reaccionado frente al hecho de publicar?. La tabla II muestra el número de publicaciones científicas realizadas en estos años. Si se tiene en cuenta las 22 publicaciones del JCR logradas en el año 1991 pueden considerarse como un inicio esperanzador las 57 publicaciones del año 1995 reflejadas en la tabla. Este número aumentó hasta alcanzar un máximo de 138 en 1998, para luego estabilizarse en 1999 a las cifras del bienio 1996-97.

El aumento espectacular de las publicaciones generadas en el año 1998 se debe a que, en ese año, se celebró el $8^{\circ}$ Congreso Nacional de Ciencía y Tecnología Metalúrgica (Mayo 1998), y los

Tabla II. Total de publicaciones por año

Table II. Total of publications per year

\begin{tabular}{cc}
\hline Año & Número \\
\hline 1995 & 57 \\
1996 & 81 \\
1997 & 84 \\
1998 & 138 \\
1999 & 83 \\
\hline
\end{tabular}

Rev. Metal. Madrid 38 (2002)

(c) Consejo Superior de Investigaciones Científicas

Licencia Creative Commons 3.0 España (by-nc) investigadores del CENIM hicieron un esfuerzo suplementario para presentar trabajos al mismo, trabajos que fueron publicados al igual que todos los presentados al Congreso, tras ser revisados por un comite científico, en un número especial de la Revista de Metalurgia (Madrid), ya para entonces incluida en el JCR. Una visión más realista de la producción científica de 1998 sería el número de 96 publicaciones en revistas del JCR sin considerar las presentadas al Congreso. Este año de 1998 destacaría como el de una mayor producción científica en los cinco años considerados.

La tabla III muestra la relación artículos/personal investigador por cada año; esta relación se mantiene en torno a 2, exceptuando 1995 (la más baja) y 1998 donde crece espectacularmente hasta más de 3 si se consideran todos los trabajos publicados en ese año, o se llega a 2,29 si no se consideran los presentados en el Congreso mencionado.

Las tablas IV y V muestran el número de autores que firman cada artículo y los artículos publicados por cada autor, respectivamente. El caracter multidisciplinar del trabajo de investigación que se realiza en el CENIM hace que sea muy díficil encontrar artículos firmados por un solo autor (Tabla IV), aunque sí se observa que esta tendencia aumenta en los dos últimos años. Con los datos presentados en esta tabla se deduce que el grueso de

Tabla III. Relación número de publicaciones/investigador por año

Table III. Number of publications/scientist ratio per year

\begin{tabular}{lc}
\hline Año & Número \\
\hline 1995 & 1,33 \\
1996 & 1,93 \\
1997 & 1,95 \\
1998 & 3,29 \\
1999 & 1,98 \\
\hline
\end{tabular}

Tabla IV. Número de autores por artículo

Table IV. Number of authors per publication

\begin{tabular}{lrrrrr}
\hline Trabajos con & 1995 & 1996 & 1997 & 1998 & 1999 \\
\hline 1 autor & 2 & - & 2 & 9 & 8 \\
2 autores & 10 & 21 & 7 & 31 & 14 \\
3 autores & 13 & 20 & 22 & 33 & 18 \\
4 autores & 20 & 24 & 34 & 42 & 23 \\
$5+$ autores & 12 & 16 & 19 & 23 & 20 \\
\hline
\end{tabular}


Tabla V. Número de artículos por autor

Table V. Number of articles per author

\begin{tabular}{lrrrrr}
\hline Autor con & 1995 & 1996 & 1997 & 1998 & 1999 \\
\hline 15 publicaciones & - & - & - & 2 & 1 \\
$10-14$ & - & 1 & 1 & 4 & - \\
$5-9$ & 4 & 11 & 9 & 12 & 9 \\
$3-4$ & 11 & 9 & 13 & 13 & 10 \\
2 & 8 & 5 & 6 & 2 & 5 \\
1 & 11 & 6 & 4 & 4 & 7 \\
\hline
\end{tabular}

los artículos publicados se han hecho contando con 3-4 autores por artículo. Un analísis más detallado de estos autores indica que estos pertenecen en muchos casos a distintos Departamentos de Investigación del CENIM e incluso a otros Centros de Investigación-Universidades-Empresas tanto nacionales como extranjeros.

En la tabla $\mathrm{V}$ se refleja el número de artículos por autor. Se observa que solo en los dos últimos años es posible encontrar autores que hayan publicado 15 o más trabajos en un año. La banda más numerosa de publicaciones por autor se situa en $3-4$, aunque seguida de cerca por la banda de 5-9 artículos.

Posteriormente se comentará cómo se distribuyen estos trabajos respecto a la posición de las revistas, en las áreas científicas consideradas, dentro del JCR.

\section{LAS REVISTAS}

Existe una regla, no escrita, en la comunidad científica según la cual solo se valora la producción científica cuando está reflejada en artículos científicos incluidos en el JCR (listado que aparece anualmente). Aunque ya se ha mencionado anteriormente, cabe señalar aquí, otra vez, que la Revista de Metalurgia (Madrid), editada por el CENIM, consiguió ser incluida en el JCR en el año 1997, hito importante en la historia de esta publicación científica ya que se podia considerar como un reconocimiento a la categoría científica de los trabajos publicados en la misma y al idioma español como medio de comunicación científico y, además, supuso un punto de satisfacción personal para no pocos investigadores del CENIM.

En el caso de los artículos publicados por el personal investigador del CENIM, estos se han publicado en una diversidad de revistas que pertenecen a un número relativamente considerable de campos o áreas científicas y pone de manifiesto la pluradidad del trabajo de investigación que se ha realizado en este Centro. Teniendo en cuenta los correspondientes JCR de los años 1995-1999, la tabla VI muestra, promediadas en el quinquenio considerado, las áreas científicas (se ha mantenido el nombre en lengua inglesa que aparece en los listados del JCR) y las revistas incluidas en las mismas en las que se han publicado trabajos por personal científico del CENIM. Como se indica en la tabla VI, ésta se ha construido considerando aquellas revistas que ocupan, para cada área científica,

Tabla VI. Áreas científicas y revistas en las que se han publicado artículos. Años: 1995-1999

Table VI. Scientific areas and journals in which articles had been published. Year: 1995-1999

\begin{tabular}{|c|c|c|c|c|c|c|}
\hline Área científica ${ }^{a}$ & TRA $^{b}$ & $\left|I^{c}\right|^{c}$ & Revista $^{d}$ & $\begin{array}{l}\text { Posición } \\
\text { en el área }\end{array}$ & $\begin{array}{c}\text { Indice } \\
\text { de impacto }\end{array}$ & $\begin{array}{c}\text { Artículos } \\
\text { publicados }^{9}\end{array}$ \\
\hline Agriculture & 102 & 1,078 & N.Z.J.Agric. Res. & 46 & 0,410 & 1 \\
\hline Chemistry & 126 & 20,228 & J.Chem.Res.(S) & 58 & 0,687 & 1 \\
\hline \multirow[t]{4}{*}{ Chemistry, Analytical } & 59 & 7,743 & Analyst & 14 & 1,843 & 1 \\
\hline & & & Anal. Chem. & 1 & 4,650 & 1 \\
\hline & & & Anal. Chim. Acta & 15 & 1,853 & 3 \\
\hline & & & Talanta & 23 & 1,228 & 1 \\
\hline \multirow[t]{4}{*}{ Chemistry, Physical } & 85 & 15,774 & J. Colloid Interface Sci. & 35 & 1,617 & 3 \\
\hline & & & Langmuir & 16 & 2,937 & 1 \\
\hline & & & Surf. Interface Anal. & 38 & 1,568 & 1 \\
\hline & & & Surf. Sci. & 22 & 2,385 & 2 \\
\hline
\end{tabular}




\begin{tabular}{|c|c|c|c|c|c|c|}
\hline Área científica ${ }^{a}$ & TRA $^{\mathrm{b}}$ & $\mid d I^{c}$ & Revista $^{d}$ & $\begin{array}{l}\text { Posición } \\
\text { en el área }\end{array}$ & $\begin{array}{c}\text { Indice } \\
\text { de impacto } f\end{array}$ & $\begin{array}{c}\text { Artículos } \\
\text { publicados }^{\mathrm{g}}\end{array}$ \\
\hline Construction \& Building Technol. & 16 & 0,777 & Cem. Concr. Res. & 1 & 0,777 & 2 \\
\hline \multirow[t]{3}{*}{ Eletrochemistry } & 10 & 7,730 & Electrochim. Acta & 4 & 1,555 & 4 \\
\hline & & & J.Appl. Electrochem. & 5 & 1,003 & 2 \\
\hline & & & J. Electroanal. Chem. & 3 & 1,663 & 2 \\
\hline \multirow[t]{2}{*}{ Energy \& Fuels } & 58 & 4,527 & Fuel & 10 & 0,841 & 2 \\
\hline & & & Sol. Energy Mater. Solar C. & 14 & 0,560 & 3 \\
\hline \multirow[t]{7}{*}{ Engineering,Chemical } & 98 & 5,037 & AIChEJ. & 5 & 1,537 & 1 \\
\hline & & & Chem. Eng. Com. & 21 & 0,782 & 1 \\
\hline & & & Chem.Eng.J. & 39 & 0,506 & 2 \\
\hline & & & Chem. Eng.Sci. & 12 & 1,024 & 1 \\
\hline & & & J.Aerosol Sci. & 3 & 1,591 & 5 \\
\hline & & & J. Chem. Technol. Biotechnol. & 21 & 0,829 & 7 \\
\hline & & & Sep.Sci.Technol. & 30 & 0,695 & 1 \\
\hline Environmental Sciences & 126 & 4,309 & Sci. Total Environ. & 40 & 1,126 & 1 \\
\hline \multirow[t]{17}{*}{ Materials Science } & 118 & 5,394 & Adv. Mater. & 3 & 3,299 & 1 \\
\hline & & & Carbon & 16 & 1,556 & 2 \\
\hline & & & Cem. Concr. Res. & 52 & 0,583 & 4 \\
\hline & & & Diam. Relat.Mater. & 13 & 1,758 & 1 \\
\hline & & & J.Adhes. Sci. Technol. & 44 & 0,713 & 1 \\
\hline & & & J.Magn. Magn. Mater. & 29 & 1,009 & 4 \\
\hline & & & J.Mater. Chem. & 10 & 1,660 & 1 \\
\hline & & & J.Mater. Res. & 17 & 1,567 & 5 \\
\hline & & & J.Mater.Sci. & 44 & 0,692 & 5 \\
\hline & & & J.Sol-Gel.Sci.Technol. & 31 & 0,966 & 2 \\
\hline & & & Mag. Concr. Res. & 66 & 0,500 & 2 \\
\hline & & & Mater. Lett. & 53 & 0,679 & 2 \\
\hline & & & Mater. Res. Bull. & 38 & 0,849 & 1 \\
\hline & & & Mater.Sci.Eng. A & 38 & 0,848 & 1 \\
\hline & & & Nanostruc. Mater. & 32 & 1,069 & 1 \\
\hline & & & Scr.Mater. & 50 & 0,645 & 2 \\
\hline & & & Thin Solid Films & 30 & 1,027 & 3 \\
\hline \multirow[t]{2}{*}{ Mat. Sci., Biomaterials } & 9 & 1,907 & Biomaterials & 3 & 1,311 & 2 \\
\hline & & & J.Biomed. Mater. Res. & 1 & 1,919 & 3 \\
\hline Mat. Sci., Ceramics & 14 & 1,524 & J.Am. Ceram. Soc. & 1 & 1,524 & 1 \\
\hline \multirow[t]{5}{*}{ Mat. Sci., Coatings \& Films } & 11 & 2,139 & J. Coat. Technol. & 7 & 0,737 & 3 \\
\hline & & & J. Electrochem. Soc. & 1 & 2,110 & 1 \\
\hline & & & J.Vac. Sci.Technol.A & 2 & 1,576 & 1 \\
\hline & & & Prog. Org. Coat. & 7 & 0,741 & 1 \\
\hline & & & Surf. Coat. Tech. & 4 & 0,895 & 6 \\
\hline Mat. Sci., Composites & & & Compos. Sci. Technol. & 3 & 0,680 & 1 \\
\hline
\end{tabular}




\begin{tabular}{|c|c|c|c|c|c|c|}
\hline Área científica ${ }^{a}$ & TRA $^{\mathrm{b}}$ & $|d|^{c}$ & Revista $^{d}$ & $\begin{array}{c}\text { Posición } \\
\text { en el área }\end{array}$ & $\begin{array}{c}\text { Indice } \\
\text { de impacto } f\end{array}$ & $\begin{array}{c}\text { Artículos } \\
\text { publicados }^{\mathrm{g}}\end{array}$ \\
\hline \multirow[t]{20}{*}{ Metallurgy \& Metallurgica Engi } & $\lg 52$ & 2,469 & Acta Metall.Mater. & 1 & 1,943 & 8 \\
\hline & & & Can. Metall. Quart. & 24 & 0,334 & 2 \\
\hline & & & Corrosion & 13 & 0,699 & 6 \\
\hline & & & Corros. Sci. & 8 & 0,900 & 18 \\
\hline & & & Hydrometallurgy & 15 & 0,954 & 24 \\
\hline & & & Intermetallics & 3 & 1,581 & 10 \\
\hline & & & Ironmaking Steelmaking & 22 & 0,378 & 3 \\
\hline & & & ISIJ INT. & 15 & 0,602 & 17 \\
\hline & & & J. Alloy Comp. & 9 & 0,880 & 2 \\
\hline & & & Mater. Corros. & 14 & 0,622 & 2 \\
\hline & & & Mater.Sci.Tech. & 11 & 0,754 & 9 \\
\hline & & & Mater.T.JIM & 8 & 0,902 & 3 \\
\hline & & & Metall. Mater. Trans. A & 8 & 0,942 & 10 \\
\hline & & & Metall. Mater. Trans. B & 14 & 0,624 & 4 \\
\hline & & & Oxid. Metal. & 3 & 1,579 & 6 \\
\hline & & & Powder Metall. & 22 & 0,327 & 1 \\
\hline & & & Scr.Mater. & 7 & 0,942 & 7 \\
\hline & & & Steel Res. & 18 & 0,448 & 5 \\
\hline & & & Werkst. Korros. & 24 & 0,376 & 4 \\
\hline & & & Z. Metallkd. & 8 & 0,878 & 3 \\
\hline Mining \& Mineral Processing & 19 & 1,201 & Miner. Eng. & 5 & 0,500 & 2 \\
\hline \multirow[t]{4}{*}{ Physics } & 64 & 12,620 & Europhys. Lett. & 8 & 2,404 & 1 \\
\hline & & & J.Phys. & 15 & 1,700 & 1 \\
\hline & & & J.Phys. A. Math.Nucl. Gen. & 17 & 1,387 & 1 \\
\hline & & & Phys. Rev. Lett. & 4 & 6,477 & 1 \\
\hline \multirow[t]{2}{*}{ Physics, Applied } & 61 & 4,319 & Appl.Phys.A & 11 & 1,538 & 1 \\
\hline & & & Philos. Mag. B & 14 & 1,489 & 1 \\
\hline \multirow[t]{2}{*}{ Physics, Condensed Matter } & 44 & 13,684 & J.Phys. Condens. Matt. & 11 & 1,479 & 1 \\
\hline & & & Phys. Rev. B & 4 & 2,909 & 2 \\
\hline \multirow[t]{3}{*}{ Spectroscopy } & 34 & 5,436 & Appl.Spectrosc. & 13 & 1,808 & 2 \\
\hline & & & J.Anal. Atom. Spectrom. & 4 & 3,292 & 6 \\
\hline & & & J.Mass. Spectrom. & 9 & 1,987 & 1 \\
\hline \multirow[t]{2}{*}{ Water Resources } & 46 & 2,084 & Water Air Soil Pollut. & 14 & 0,971 & 1 \\
\hline & & & Water Res. & 3 & 1,616 & 1 \\
\hline
\end{tabular}

\footnotetext{
a Se consideran las áreas científicas en las que están incluidas revistas en las que se han publicado trabajos y están situadas en una posición igual o superior al $50 \%$ de las revistas del área.

${ }^{\mathrm{b}}$ Total revistas en el área.

c Índice de impacto de la revista situada en primer lugar de cada área.

$b, c, e, f$ Valores promedio de los años considerados, teniendo en cuenta el punto ${ }^{a}$.

${ }^{d}$ Se consideran las revistas que se encuentran situadas en una posición igual o superior al $50 \%$ del total de revistas en cada área.

${ }^{g}$ Artículos publicados en los que ha participado algún miembro del personal científico (según Tabla I) del CENIM.
} 
una posición igual o superior al $50 \%$ del total de las revistas incluidas en cada área.

La tabla VII muestra un resúmen de los resultados mostrados en la tabla anterior. En esta tabla se observa que, a lo largo del período de tiempo considerado, el total de áreas científicas en las que se han publicado trabajos en revistas incluidas en el JCR ronda las $16\left(\mathrm{~A}_{\mathrm{TOT}}\right)$, aunque este número disminuye si se consideran las áreas que incluyen revistas en posiciones iguales o superiores al $50 \%$ $\left(A_{50}\right)$.

El número total de revistas incluidas en el JCR en las que se ha publicado, es de 44. En los tres primeros años considerados (1995-1997), el tanto por ciento de estas revistas que se situaban en una posición dentro del $50 \%\left(\mathrm{R}_{50}\right)$ en las áreas correspondientes, era del $75 \%$ del total de las revistas, pasando a cerca de un 80 \% para los años 19981999. Este crecimiento es más espectacular si se restrigen las revistas a posiciones situadas por encima del $25 \%\left(\mathrm{R}_{25}\right)$ del total de revistas en las áreas. Así, en el tramo 1995-1997, cerca de un 34 \% de las mismas se situaban dentro de este porcentaje, para crecer a un $49 \%$ y a un $59 \%$ para los años 1998 y 1999 respectivamente.

La distribución de los trabajos publicados en estas revistas sigue una línea parecida, aumentando del $70 \%$ (1997) al $80 \%$ (1999) el porcentaje de trabajos publicados en revistas posicionadas en un puesto igual o superior al $50 \%\left(\mathrm{~T}_{50}\right)$ del total de cada área. En el caso de considerar las revistas situadas en la banda del $25 \%\left(\mathrm{~T}_{25}\right)$, considerando como en los casos anteriores una posición igual o
Tabla VII. Resumen de los datos de publicaciones presentados en la tabla VI

Table VII. Summary of data shown in table VI

\begin{tabular}{lcccccc}
\hline Año & & 1995 & 1996 & 1997 & 1998 & 1999 \\
\hline Áreas científicas & $\mathrm{A}_{\text {TOT }}$ & 17 & 13 & 15 & 15 & 17 \\
\multirow{4}{*}{ Revistas } & $\mathrm{A}_{50}$ & 14 & 11 & 11 & 13 & 14 \\
& $\mathrm{R}_{\text {TOT }}$ & 42 & 41 & 46 & 49 & 44 \\
& $\mathrm{R}_{25}$ & 13 & 17 & 16 & 24 & 26 \\
\multirow{4}{*}{ Trabajos } & $\mathrm{R}_{50}$ & 32 & 28 & 33 & 38 & 34 \\
& $\mathrm{~T}_{\text {TOT }}$ & 57 & 81 & 84 & $96^{*}$ & 83 \\
& $\mathrm{~T}_{25}$ & 16 & 23 & 21 & 48 & 56 \\
& $\mathrm{~T}_{50}$ & 46 & 55 & 59 & 73 & 64 \\
\hline
\end{tabular}

"Excluidos los trabajos presentados al 8 Congreso Nacional de Ciencia y Tecnología Metalúrgicas.

superior a este porcentaje, en los años 1995-1997 el porcentaje de trabajos publicados en las revistas fué de cerca del $28 \%$, para incrementarse a un $50 \%$ en 1998 y a un $68 \%$ en 1999.

Desde un punto de vista global, la tabla VIII muestra las revistas en las que se ha publicado algún artículo y que presentan los díez mayores valores del índice de impacto. Se observa que, entre éstas, cinco son revistas incluidas en las áreas de Química Analítica o Espectroscopía (con un fuerte componente analítico en este caso partícular). En cuanto a la posición relativa que ocupan

Tabla VIII. Las revistas con los diez valores mayores del índice de impacto en las que personal investigador del CENIM ha publicado artículos en el quinquenio 1995-99

Table VIII. The top ten highest-impact journals in which articles had ever been published

\begin{tabular}{lllcrc}
\hline Puesto & Revista & Año & Índice de impacto & Citas & Posición* \\
\hline 1 & Phys. Rev. Lett. & 1996 & 6,477 & 132.197 & $4 / 62$ \\
2 & Anal. Chem. & 1996 & 4,650 & 37.777 & $1 / 52$ \\
3 & J.Anal. Atom. Spectrom. & 1998 & 3,845 & 4.582 & $4 / 35$ \\
4 & J.Anal. Atom. Spectrom. & 1999 & 3,677 & 5.087 & $3 / 34$ \\
5 & J.Anal. Atom. Spectrom. & 1997 & 3,595 & 4.166 & $3 / 34$ \\
6 & Adv. Mater. & 1995 & 3,299 & 2.327 & $3 / 88$ \\
7 & Phys. Rev. B & 1996 & 2,975 & 130.789 & $4 / 40$ \\
8 & Langmuir & 1999 & 2,937 & 210.721 & $16 / 89$ \\
9 & Phys. Rev. B & 1998 & 2,842 & 134.476 & $4 / 48$ \\
10 & J.Anal. Atom. Spectrom. & 1995 & 2,797 & 3.288 & $5 / 33$ \\
\hline
\end{tabular}

*Puesto de la revista/número total de revista en el área. 
esas diez revistas, todas están situadas por encima del $25 \%$ del total de las revistas consideradas en cada área, dado que cada una de estas categorias del JCR tiene su propia clasificación respecto al indice de impacto.

\section{CONCLUSIONES}

Los datos correspondientes al quinquenio 19951999 permiten asegurar que el personal investigador del CENIM respondió de una manera efectiva, tanto de forma cuantitativa como cualitativa, al reto de las publicaciones científicas que lanzó la Comisión de Evaluación.

\section{REFERENCIAS}

[1] Memorias del Centro Nacional de Investigaciones Metalúrgicas de los años 1995-1999, Madrid, España.

[2] Journal Citation Reports de los años 1995-1999, publicación del Institute for Scientific Information, Filadelfia, USA. 